Supporting information

\title{
Unique Thermoresponsive Polymer Micelle Behavior via Cooperative Polymer Corona Phase Transitions
}

\author{
Masamichi Nakayama and Teruo Okano * \\ Institute of Advanced Biomedical Engineering and Science, \\ Tokyo Women's Medical University, \\ 8-1 Kawada-cho, Shinjuku-ku, Tokyo 162-8666, Japan \\ e-mail address: tokano@abmes.twmu.ac.jp
}

*Corresponding author.

\section{General RAFT Polymerization}

A chain transfer agent (CTA), 2-cyanopropyl dithiobenzoate (CPDB), was prepared by modification of previous methods $[1,2]$. Benzyl methacrylate (BzMA, 0.03 mol), CPDB (3.60 mmol), and 2,2'-azobisisobutyronitrile (AIBN, $0.72 \mathrm{mmol}$ ) were dissolved in $60 \mathrm{ml}$ benzene. The solution was degassed under reduced pressure by triplicate freeze-pump-thaw cycles, and polymerization was carried out at $70^{\circ} \mathrm{C}$ for $7 \mathrm{~h}$. After polymerization, polymers were precipitated in an excess of methanol and purified by repeated precipitations, followed by thorough drying under vacuum (yield 53\%). 
Diblock copolymers with thermoresponsive segments were synthesized using characterized PBzMA as the macro-CTA. $N$-Isopropylacrylamide (IPAAm, $6.5 \mathrm{mmol}$ ), $N, N$-dimethylacrylamide (DMAAm, $3.5 \mathrm{mmol})$, AIBN (0.02 mmol), and PBzMA (0.1 mmol) were dissolved in $5 \mathrm{ml}$ benzene), and solutions were degassed by freeze-pump-thaw cycles for three times. Polymerization was then carried out at $70^{\circ} \mathrm{C}$ for $22 \mathrm{~h}$. After polymerization, polymer products were obtained by precipitation twice into an excess amount of diethyl ether, and then dried in vacuo (yield 44\%).

Number-averaged molecular weights $(\mathrm{Mn})$ of the obtained polymers were estimated by a ${ }^{1} \mathrm{H}-\mathrm{NMR}$ spectrometer $\left(400 \mathrm{MHz}\right.$, Varian Inc., CA) using dimethyl sulfoxide- $\mathrm{d}_{6}$ (Aldrich) as a solvent. Mn of PBzMA was determined from the number of monomer units in PBzMA estimated from the areas of ortho-phenyl (7.9 ppm) and methylene peaks (4.9 ppm) corresponding to terminal dithiobenzoate groups and BzMA side chains, respectively. Mn and monomer composition of IPAAm, DMAAm and BzMA in the diblock copolymers were determined by the proton peak areas of methine of IPAAm (3.9 ppm), methyl of DMAAm (2.8 ppm) and methylene (4.9 ppm) of BzMA, respectively. Polydispersities of the polymers were determined by gel permeation chromatography (Tosoh, SC-8020 with GPC column: TSK gel G2000H $\mathrm{HR}_{\mathrm{H}}, \mathrm{G} 3000 \mathrm{H}_{\mathrm{HR}}, \mathrm{G} 4000 \mathrm{H}_{\mathrm{HR}}$, Tokyo, Japan, calibrated with polystyrene standards, elution rate: $1.0 \mathrm{ml} / \mathrm{min}$ ) at $45^{\circ} \mathrm{C}$ using DMF containing $10 \mathrm{mM} \mathrm{LiCl}$ as an eluent.

\section{Aminolysis and Conversion of Polymer Terminal Groups}

The obtained polymers $(300 \mathrm{mg})$ were dissolved in $2 \mathrm{ml}$ deoxidized $N, N$-dimethylformamide by $\mathrm{N}_{2}$ gas bubbling for $1 \mathrm{~h}$ prior to use. 2 -Ethanolamine $(0.65$ 
mmol) in $1 \mathrm{ml}$ DMF was then added to polymer solutions. After 5 minutes, iodoethanol $(1.3 \mathrm{mmol})$ in $1 \mathrm{ml} \mathrm{DMF}$ were added to the reaction solutions, followed by reaction at $40^{\circ} \mathrm{C}$ for $15 \mathrm{~h}$ under a nitrogen atmosphere. Reaction solutions were then dialyzed against distilled water at $5^{\circ} \mathrm{C}$ for $48 \mathrm{~h}$ (MWCO 1000, Spectra/Por 6, Spectrum Medical Industries, CA), and polymers were recovered by freeze-drying.

\section{Preparation of Polymeric Micelles}

Formation of micelles was simultaneously performed by a dialysis method. Amphiphilic diblock copolymers were dissolved in $N, N$-dimethylacetamide, and solutions were then dialyzed against distilled water at $5^{\circ} \mathrm{C}$ for $24 \mathrm{~h}$ using a dialyzer with a Spectra/Por 6 membrane (MWCO: 1000).

\section{Dynamic Light Scattering Measurements}

Hydrodynamic mean diameters and size distributions of polymeric micelles were determined by dynamic light scattering (DLS) using a Zetasizer Nano ZS (Malvern Instruments, Ltd., UK). Concentration of all polymer samples was $1 \mathrm{mg} / \mathrm{ml}$ in distilled water. All measurements were performed at $20^{\circ} \mathrm{C}$.

\section{Fluorescence Measurements}

Fluorescence spectra were obtained using an FP-6500 spectrofluorimeter (JASCO, Tokyo, Japan) with pyrene as a hydrophobic fluorescent probe. Pyrene dissolved in acetone $(480 \mu \mathrm{M}, 5 \mu \mathrm{l})$ was added to $4 \mathrm{ml}$ of each polymer solution at various concentrations. Sample solutions were kept at $20^{\circ} \mathrm{C}$ for 2 days before measurements 
were conducted to allow for complete evaporation of acetone. Fluorescence excitation was carried out at $370 \mathrm{~nm}$ and excitation spectra were recorded from 300 to $360 \mathrm{~nm}$. The ratio of the intensities at 337 and $334 \mathrm{~nm}$ were then analyzed to determine critical micelle concentrations (CMC) of each micelle. CMC values were defined as the intersection of the lines drawn through the points of flat regions at low polymer concentration and the drastically increasing regions at high concentration in the $I_{337} / I_{334}$ plot against the logarithm of polymer concentration [3].

\section{Estimation of Phase Transition Behavior}

Changes in absorbance $(0.5 \mathrm{mg} / \mathrm{ml}$ polymers in Dulbecco's Phosphate buffered saline without calcium chloride and magnesium chloride (DPBS(-)) and in optical transmittance $(10 \mathrm{mg} / \mathrm{ml}$ polymers in DPBS(-)) of polymeric micelles in DPBS(-) with temperature were measured at 600nm using a UV-vis spectrometer (JASCO UV-530) with the heating rate at $0.1^{\circ} \mathrm{C} / \mathrm{min}$. A sample cell was thermostated with a Peltier-effect cell holder (EHC-477T, JASCO). Lower critical solution temperature (LCST) of the polymer solutions was defined as a temperature at which $50 \%$-decrease in optical transmittance.

\section{NMR studies for the diblock copolymers [4]}


(a) PID-b-PBzMA in DMSO-d6
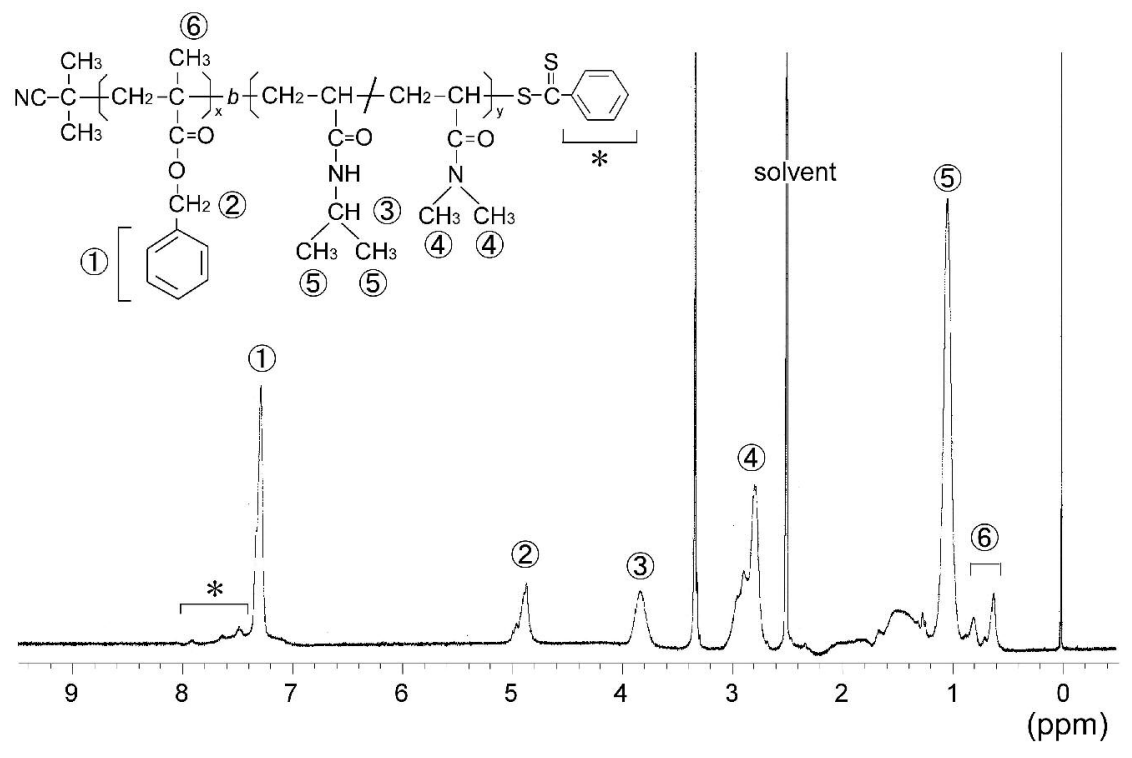

(b) PID/PBzMA micelle in $\mathrm{D}_{2} \mathrm{O}$

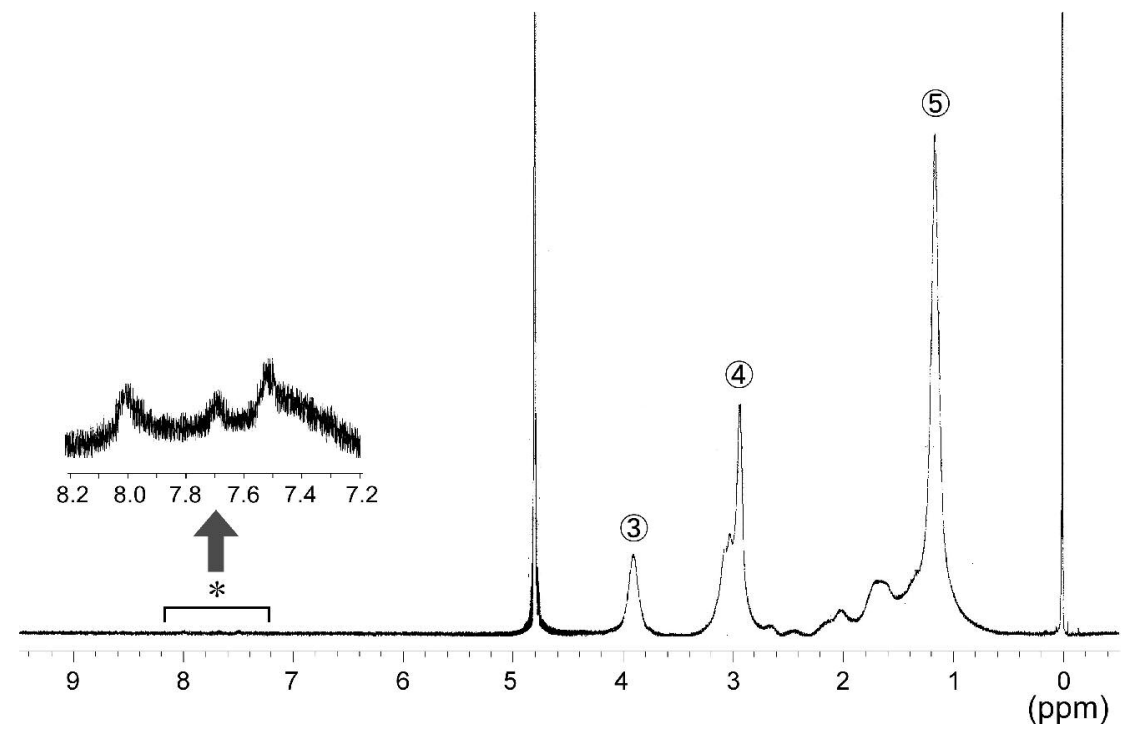

Figure S1. ${ }^{1} \mathrm{H}-\mathrm{NMR}$ spectra $\left(400 \mathrm{MHz}, 20^{\circ} \mathrm{C}\right)$ of (a) block copolymer phenyl-terminated PID- $b$-PBzMA in DMSO- $\mathrm{d}_{6}$, and (b) M (Phe) micelles composed of phenyl-terminated PID- $b$-PBzMA in $\mathrm{D}_{2} \mathrm{O}$. 
In DMSO- $\mathrm{d}_{6}$, where micellar formation is not expected, all NMR resonances attributed to IPAAm, DMAAm, and BzMA units were detected as shown in Figure S1(a). However, the NMR spectrum in $\mathrm{D}_{2} \mathrm{O}$ showed complete loss of BzMA resonances (0.6, $0.8,4.9$, and $7.3 \mathrm{ppm}$, respectively) due to suppressed molecular motion of the aggregated hydrophobic chains (Figure S1(b)). ${ }^{1} \mathrm{H}-\mathrm{NMR}$ study in aqueous solution showed mainly hydrophilic PID signals and complete loss of PBzMA resonance signals, strongly indicative of a stable core-shell micellar formation with a highly viscous inner core. Proton signals for hydrophobic phenyl groups located at corona-forming polymer termini were also observed in aqueous micelle solution. This suggests that terminal hydrophobic phenyl groups did not interact with or partition into the micelle inner cores due to highly hydrated PID chains existing as extended conformations.

\section{References}

(1) Thang, S. H.; Chong, (B) Y. K.; Mayadunne, R. T. A.; Moad, G.; Rizzardo, E. Tetrahedron Lett. 1999, 40, 2435-2438.

(2) Mitsukami, Y.; Donovan, M. S.; Lowe, A. B.; McCormick, C. L. Macromolecules 2001, 34, 2248-2256.

(3) Zhao, C.; Wang, Y.; Winnik, M. A.; Riess, G.; Croucher, M. D. Langmuir 1990, 6, 514-516.

(4) Nakayama, M.; Okano T. Biomacromolecules 2005, 6, 2320-2327. 